\title{
Diagnostic Accuracy of D-Dimer Testing and the Revised Geneva Score in the Prediction of Pulmonary Embolism
}

This article was published in the following Dove Press journal: International Journal of General Medicine

\author{
Mostafa A Abolfotouh (D) ${ }^{1-3}$ \\ Khaled Almadani ${ }^{3}$ \\ Mohammed A Al Rowaily (iD) 2,3 \\ 'King Abdullah International Medical \\ Research Center, Riyadh, Saudi Arabia; \\ ${ }^{2}$ King Saud Bin-Abdulaziz University for \\ Health Sciences, Riyadh, Saudi Arabia; \\ ${ }^{3}$ King Abdulaziz Medical City, Ministry of \\ National Guard-Health Affairs, Riyadh, \\ Saudi Arabia
}

Background: Pulmonary embolism (PE) diagnosis can sometimes be challenging due to the disease having nonspecific signs and symptoms at the time of presentation. The present study aimed to evaluate the validity of the D-dimer in combination with the revised Geneva score (RGS) in the prediction of pulmonary embolism.

Patients and Methods: This is a retrospective study of 2010 patients with suspected PE who had undergone both D-dimer testing followed by chest CT angiography (CTPA), irrespective of the D-dimer test results, at King Abdulaziz Medical City, Riyadh, Saudi Arabia, over 3 years, from Jan. 2016 to Jan. 2019. The predictive accuracy of D-dimer, adjusted D-dimer, and RGS was calculated. The receiver operating characteristic "ROC" curve was applied to allocate the optimum RGS cutoff for PE prediction.

Results: The overall prevalence of PE was $16 \%$. It was $0 \%, 25.8 \%$, and $88.9 \%$ in low, intermediate, and high clinical probability categories of RGS, respectively. Both conventional and age-adjusted D-dimer thresholds showed significant level of agreement (kappa $=0.81, \mathrm{p}<0.001)$, high sensitivity ( $94 \%$ and $92.8 \%$ ), high negative predictive value "NPV" (91.2\% and 91.4\%), low specificity (12.3\% and 15.3\%), and low positive predictive value "PPV" (17.5\% and 17.8\%), respectively. Combination of the age-adjusted D-dimer threshold and RGS at a cut-off of 5 points would provide $100 \%$ sensitivity and $61.7 \%$ specificity $34.1 \% \mathrm{PPV}, 100 \% \mathrm{NPV}$, and 0.87 area under the curve "AUC". At an RGS cutoff $<5$ points, PE could have been ruled out in more than one-half $(1036,51.5 \%)$ of all suspected cases, and would have saved the cost of CTPA.

Conclusion: Conventional and age-adjusted D-dimer tests showed high levels of agreement in the prediction of PE, high sensitivity, and low specificity. RGS has a good performance in PE prediction. Using the revised Geneva score alone rules out PE for more than one-half of all suspected without further imaging.

Keywords: validity, sensitivity, specificity, deep vein thrombosis, agreement, clinical probability, D-dimer, age-adjusted threshold, CTPA

\section{Introduction}

Pulmonary embolism (PE) is a disease that is commonly encountered in the emergency department. ${ }^{1-3}$ The incidence of PE is estimated to be 60-70 per 100,000 general population. ${ }^{4}$ In Saudi Arabia, the incidence of Venous Thromboembolism (VTE) comprised of PE and Deep Vein Thrombosis (DVT) is estimated to be approximately 100 per 100,000 per year, with an estimated number of 25,000 patients per year. ${ }^{2}$ Its diagnosis can sometimes be challenging due to the disease having nonspecific signs and symptoms at the time of presentation. ${ }^{5}$ The
Correspondence: Mostafa A Abolfotouh Research Center (Mail Code 3533), King Saud Bin-Abdulaziz University for Health Sciences (KSAU-HS), King Abdulaziz Medical City, National Guard Health Affairs, POB 22490, Riyadh I I426, Saudi Arabia

Email mabolfotouh@gmail.com
International Journal of General Medicine 2020:13 |537-1543 
diagnosis can be made clinically using some validated risk assessment tools such as the Revised Geneva Score ${ }^{6}$ and the Wells score ${ }^{7}$ based on the signs and symptoms of the patient. After that, the next step would be obtaining a serum D-dimer level from the patient. ${ }^{8} \mathrm{~A}$ negative result would rule out the possibility of having PE and would subsequently eliminate the need to use further confirmation by chest CT angiography (CTAP).

D-dimer levels are affected by age ${ }^{9,10}$ and many other variables such as malignancies and sepsis. ${ }^{9}$ This usually leads to unnecessary exposure to radiation while undergoing CTPA with studies showing that only $20 \%$ of those show positive results. ${ }^{11}$ The overuse of CTPA $^{12}$ has been discussed in studies before highlighting that it has many disadvantages such as its effect on the patients. A study has shown that a single CTPA was associated with an average lifetime risk for cancer of 57/100,000 for females aged 17-19 years to 8/100,000 for males and females aged 80-89 years. ${ }^{13}$ Furthermore, the use of CPTA holds up the efficacy and the flow of the ER by taking up beds for an unnecessary amount of time.

Many studies have concluded that the use of the ageadjusted D-dimer level in patients $>50$ years old with low to moderate clinical probability to develop PE is safe and reduces the need to use unnecessary CTPA. ${ }^{14-16}$ The use of age-adjusted D-dimer cutoff value (patient's age x 10 $\mu \mathrm{g} / \mathrm{L})$. It has been shown that patients $(42 \%)$ aged more than 50 years old with low clinical probability had PE excluded in comparison with patients (36\%) when fixed D-dimer cutoff value $(500 \mu \mathrm{g} / \mathrm{L})$ was applied. ${ }^{13}$ A case report with a patient older than 50 years who had an ageadjusted d-dimer level below the threshold and tested positive for $\mathrm{PE}$, later on, has raised a point regarding the possibility of a false-negative result. ${ }^{17}$ One research compared the age-adjusted D-dimer level with a Clinical Probability Adjusted D-dimer level (1000 $\mu \mathrm{g} / \mathrm{L}$ for low probability and $500 \mu \mathrm{g} / \mathrm{L}$ if intermediate), with a conclusion that the age-adjusted test is the preferred choice since it had a better Negative predictive value. ${ }^{18}$

No available guidelines are yet available at KAMC for the management of patients with suspected PE, and every suspected patient is subjected to both D-Dimer testing followed by CTPA, without waiting for the D-Dimer results that would take 4 to $5 \mathrm{~h}$ to be released, and this would lead to unnecessary exposure of patients to radiation. Thus, the aims of this study were (1) to compare the diagnostic accuracy of conventional D-dimer level with that of the age-adjusted D-dimer level, (2) to determine the diagnostic accuracy of using the age-adjusted D-dimer level, in combination with the revised Geneva score, for prediction and/or exclusion of $\mathrm{PE}$, and (3) to estimate the amount of unnecessary exposure of patients to radiation, in adults who underwent CTPA in the past 3 years in King Abdulaziz Medical City, Riyadh, Saudi Arabia. The results of this study would help in writing the policy and procedures of management of patients with suspected PE in our health facility.

\section{Patients and Methods}

This is a retrospective study of 2010 patients with suspected PE (Those presenting with symptoms suggestive of PE including, physical exam, vital signs, and risk factors) who had undergone both D-dimer testing followed by chest CT angiography (CTPA) over 3 years, from January 2016 to January 2019. Patients who underwent CT angiography with no D-dimer test results were excluded from the study. Patients' charts from hospital records (Best-Care) were reviewed and information was extracted using a data collection sheet. The study was conducted at the Emergency Care Center (ECC), King Abdulaziz Medical City (KAMC) in the central region of Saudi Arabia. KAMC is the largest health institution of the Ministry of National Guard Health Affairs (MNG-HA). MNG-HA provides healthcare services to national guard service members and their dependents through large medical cities located in the three most densely populated regions of KSA, namely the Central, Western, and Eastern regions. All facilities have been Joint Commission International (JCI) accredited since 2006.

ECC provides services for a rapidly growing patient population in all of its catchment areas, and it is considered the largest emergency care in Riyadh with a capacity of 125 beds and an ability to be increased if needed. The center has been established to provide urgent care services for all patients mainly trauma and critical medical cases. The center contains trauma X-Ray and Stat Lab to be ready when time becomes critical. It is divided into two main sections; Adult care (30 consultants and 34 Staff Physicians) and Pediatric care (20 consultants and 21 Staff Physicians). The average number of ECC visits is about 700 patients per day with some seasonal variations. Access to emergency services is determined by a process of ED triage. At triage, nurses assign an urgency rating according to observable physiological parameters.15 ECC follows the Canadian ED Triage and Acuity Scale (CTAS) 
guidelines in categorizing cases, based on the severity of the cases.

All patients, Saudis, and non-Saudis, ages 18 years or more, of both sexes were included. The following data were collected: Gender, D-dimer level, Result of the Chest CT Angiography, and elements of the Revised Geneva Score (age, previous PE/DVT, surgery/lower limb or fracture in the past month, having active malignancy, unilateral lower limb pain, hemoptysis, Heart rate, and pain on limb palpation). ${ }^{19}$ The score was calculated after reviewing each patient file including the physician notes and the flow chart of the vital signs. There was no specific form for the RGS in our system.

\section{Operational Definitions}

Acute PE refers to obstruction of the pulmonary artery or one of its branches by material (eg, thrombus, tumor, air, or fat) that originated elsewhere in the body. The diagnosis was based upon a $\mathrm{CT}$ angiogram report from the radiology department confirming that a thrombus was the main cause of the obstruction. A value of D-dimer above 500 FEU $(0.5 \mu \mathrm{g} / \mathrm{mL})$ was considered the cutoff value above which imaging modality was mandated. Age-adjusted D-Dimer threshold was calculated by multiplying the age of the patient 50 years and above by 10 [Age-adjusted D-dimer (FEU) =Patient's age $\mathrm{x} 10)$ or $[$ Age-adjusted D-dimer $(\mu \mathrm{g} / \mathrm{mL})=($ Patient's age $\mathrm{x} 10) / 1000]$. The manufacturer of the D-dimer test at our facility is SIEMENS [Instrument: Sysmex CS-2000i/ CS-2100i Systems].

\section{Data Analysis}

Data were entered and analyzed using SPSS software version 26. Quantitative variables were expressed as means and standard deviation. Categorical values were expressed as frequencies and percentages. Sensitivity, Specificity, NPV, and PPV were calculated for the diagnostic test results. The level of agreement between conventional and age-adjusted D-dimer was calculated with kappa. Estimation of the amount of unnecessary radiation exposure was done by calculation of the percentage of patients with positive age-adjusted D-Dimer and did not have PE by CTPA. Receiver Operator Characteristic curve was applied to allocate the optimum RGS cutoff for the exclusion of PE. Significance was considered at a $p$-value of $<0.05$.

\section{Results}

Of 2010 patients with an average age of $52.2 \pm 20.2$ years, two-thirds $(65.1 \%)$ were females, with the previous history of; DVT or PE (2\%), surgery and/or fracture of lower limb $(6.9 \%)$, active malignant conditions $(14.4 \%)$, unilateral lower limb pain $(0.6 \%)$, and hemoptysis $(0.7 \%)$ (Table 1). A total of 332 (16.5\%) patients were diagnosed as PE patients by CTPA. The sensitivity of conventional D-dimer for PE was high (94\%), with only 20 false negatives $(1.0 \%)$, decreasing slightly to $92.8 \%$ with ageadjusted D-dimer testing, with 24 (1.2\%) false negatives. The specificity of D-dimer for PE was low by the conventional threshold $(12.3 \%)$, increasing to $15.3 \%$ by the ageadjusted threshold, Table 2. False-negative rates were $1.0 \%$ and $1.2 \%$ for the conventional and age-adjusted thresholds, respectively.

The level of agreement between conventional D-dimer and age-adjusted D-dimer testing in prediction of $\mathrm{PE}$ was high $[(976+98) / 1128) \quad \mathrm{x} \quad 100=95.2 \%, \quad \mathrm{kappa}=0.081$, $\mathrm{p}<0.001]$. Both thresholds agree on $100 \%[(8+90) /(8$ $+90)]$ of negative cases, and 97.3\% [(181+795)/(185 $+845)$ ] of positive cases. Agreement in Positive CTPA is $95.5 \%$ [(181+8)/198], and agreement in negative CTPA is $94.7 \%$ [(795+90)/935]. In total, 98 patients had a D-dimer

Table I Personal and Clinical Characteristics of the Study Sample

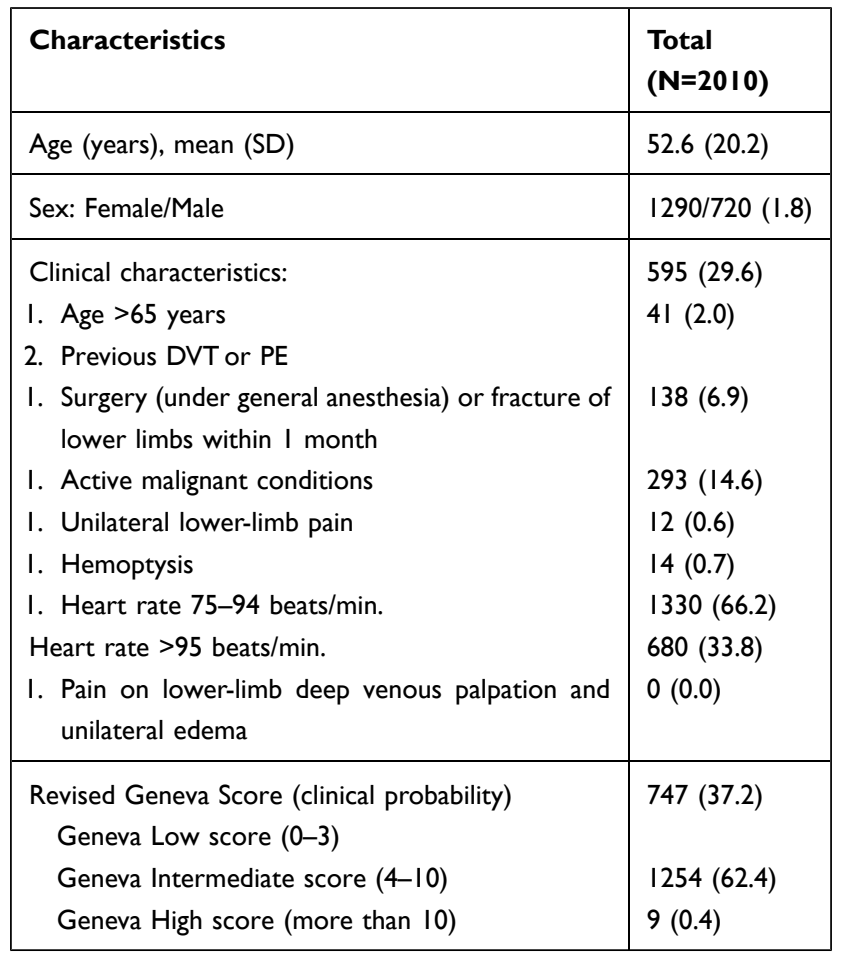

Abbreviations: DVT, deep vein thrombosis; PE, pulmonary embolism. 
Table 2 Comparison Between Conventional D-Dimer and Age-Adjusted D-Dimer in Accuracy of Prediction of Pulmonary Embolism

\begin{tabular}{|c|c|c|c|}
\hline & \multicolumn{2}{|l|}{ Chest CT Scan } & \multirow[b]{2}{*}{ Total No.(\%) } \\
\hline & Positive No.(\%) & Negative No.(\%) & \\
\hline \multicolumn{4}{|l|}{ Conventional D-dimer } \\
\hline Abnormal & $312(15.5)$ & $1472(73.2)$ & $1784(88.8)$ \\
\hline Normal & $20(1.0)$ & $206(10.2)$ & $226(11.2)$ \\
\hline Total & $332(16.5)$ & $1678(83.5)$ & 2010 \\
\hline \multicolumn{4}{|c|}{ Sensitivity $=94 \%$, specificity $=12.3 \%, P P V=17.5 \%, N P V=91.2$, kappa $=0.009, p=0.001$} \\
\hline \multicolumn{4}{|l|}{ Age-adjusted D-dimer } \\
\hline Abnormal & $308(15.3)$ & $1422(70.7)$ & $1730(86.1)$ \\
\hline Normal & $24(1.2)$ & $256(12.7)$ & $280(13.9)$ \\
\hline Total & $332(16.5)$ & $1678(83.5)$ & 2010 \\
\hline
\end{tabular}

Abbreviations: PPV, positive predictive value; NPV, negative predictive value.

below the conventional threshold of $0.5 \mathrm{Un}$, and $8 \mathrm{PE}$ patients of them $(8.2 \%)$ were missed. Meanwhile, 152 patients had a D-dimer below the age-adjusted threshold, 12 PE patients were missed (7.9\%), Table 3.

According to the RGS, 747 patients (37.2\%) had a low clinical probability, $1254(62.4 \%)$ an intermediate clinical probability, and $9(0.4 \%)$ a high clinical probability (Table 3). The prevalence of PE was $0 \%, 25.8 \%$, and $88.9 \%$ in these three different probability groups, respectively, Table 4 . The receiver operating characteristic curve was applied for the allocation of an optimum RGS cut-off for the prediction of PE (Figure 1). The cutoff $\geq 5$ points was the optimum RGS cut-off for prediction of PE, with $100 \%$ sensitivity, $61.7 \%$ specificity, $34.1 \% \mathrm{PPV}$, and $100 \% \mathrm{NPV}$, and an area under the curve of 0.87 , Table 4.

$\mathrm{PE}$ could have been ruled out for more than one-half $(51.5 \%)$ of all suspected cases $(870+166=1036)$ if they had used the Geneva score alone, Table 5.

\section{Discussion}

In this study, the prevalence of PE was $16.5 \%$ among patients with suspected PE. This prevalence was comparable with an incidence of $16 \%$ in a previous study by Klok et $\mathrm{al}^{20}$ and it ranged from $10.1 \%$ to $20.7 \%$ in other studies. ${ }^{13,21,22}$ Clinical assessment of the probability of $\mathrm{PE}$ is important in the diagnostic approach of PE. Applying a clinical decision rule in combination with the age-adjusted D-dimer threshold would have raised the number of patients in whom PE could have been ruled out without further imaging. According to the RGS, 747 patients (37.2\%) had a low clinical probability, 1254 $(62.4 \%)$ an intermediate clinical probability, and 9 $(0.4 \%)$ a high clinical probability (Table 3$)$. The prevalence of PE was $16.5 \%, 0 \%, 25.8 \%$, and $88.9 \%$ in these three different probability groups, respectively. The corresponding prevalence figures were; $9 \%, 30 \%$, and $68 \%$ as reported in the Postoperative Investigation of Pulmonary Embolism Diagnosis (PIOPED) study, ${ }^{23}$ and $8.3 \%$ 22.8\%,

Table 3 Performance of D-dimer and Age-Adjusted D-dimer Testing and Their Level of Agreement in the Prediction of Pulmonary Embolism

\begin{tabular}{|c|c|c|c|c|c|c|}
\hline \multirow{3}{*}{ Conventional D-Dimer } & \multicolumn{4}{|c|}{ Age-Adjusted D-dimer } & & \\
\hline & \multicolumn{2}{|c|}{ Abnormal } & \multicolumn{2}{|c|}{ Normal } & \multicolumn{2}{|l|}{ Total } \\
\hline & $\mathrm{n}$. & PE & n. & PE & $\mathrm{n}$. & PE \\
\hline Abnormal & 976 & 181 & 54 & 4 & 1030 & $185(18.0)$ \\
\hline Normal & o & 0 & 98 & 8 & 98 & $8(8.2)$ \\
\hline Total & 976 & $|8|(\mid 8.5)$ & 152 & $12(7.9)$ & 1128 & $193(17.1)$ \\
\hline
\end{tabular}

Notes: kappa $=0.08, \mathrm{p}<0.00 \mathrm{I} . \mathrm{I}$; Level of agreement, $[(976+98) / 1 / 28) \times 100=95.2 \%$; Agreement on negative cases, $[(8+90) /(8+90)]=100 \%$; Agreement on positive cases, $[(181+795) /(185+845)]=97.3 \%$; Agreement in Positive CTPA, $[(181+8) / / 98=95.5 \%$; Agreement in negative CTPA, $[(795+90) / 935]=94.7 \%$.

Abbreviation: PE, pulmonary embolism. 
Table 4 Prevalence of Pulmonary Embolism in Patients with Different Revised Geneva Score Categories and Validity of a Cutoff of 5 Revised Geneva Score $\geq 5$ in the Prediction of Pulmonary Embolism

\begin{tabular}{|c|c|c|c|}
\hline \multirow{2}{*}{$\begin{array}{l}\text { Revised Geneva Score } \\
\text { ( } 3 \text { categories) }\end{array}$} & \multicolumn{2}{|l|}{ Chest CT Scan } & \multirow[b]{2}{*}{ Total no.(\%) } \\
\hline & Positive no.(\%) & Negative no.(\%) & \\
\hline Low & $0(0.0)$ & $747(100.0)$ & 747 (37.2) \\
\hline Intermediate & $324(25.8)$ & $930(74.2)$ & $1254(62.4)$ \\
\hline High & $8(88.9)$ & $\mathrm{I}(\mathrm{II} . \mathrm{I})$ & $9(0.4)$ \\
\hline Total & $332(16.5)$ & $1678(83.5)$ & $2010(100.0)$ \\
\hline \multirow{2}{*}{$\begin{array}{l}\text { Revised Geneva Score } \\
\text { ( } 2 \text { categories) }\end{array}$} & \multicolumn{2}{|l|}{ Chest CT Scan } & \\
\hline & Positive no.(\%) & Negative no.(\%) & Total no.(\%) \\
\hline Likely (5 points or more) & $332(100.0)$ & $642(38.3)$ & $974(48.5)$ \\
\hline Unlikely (<5 points) & $0(0.0)$ & $1036(61.7)$ & $1036(51.5)$ \\
\hline Total & 332 & 1678 & 2010 \\
\hline
\end{tabular}

Notes: Sensitivity=100\%; specificity= 61.7\%; PPV=34.1\%; NPV= 100; kappa $=0.35, \mathrm{p}=<0.001$.

and $71.4 \%$ as reported by Klok et $\mathrm{al}^{20}$ who concluded that PE could be excluded in patients with a low or intermediate clinical probability by RGS and a normal D-dimer level. However, from the results of our study, it may be safe to exclude PE in patients by a low clinical probability by the revised Geneva score irrespective of D-dimer results.

ROC curve was applied to allocate the optimum RGS cutoff for PE exclusion. It showed the cutoff of $>5$ points

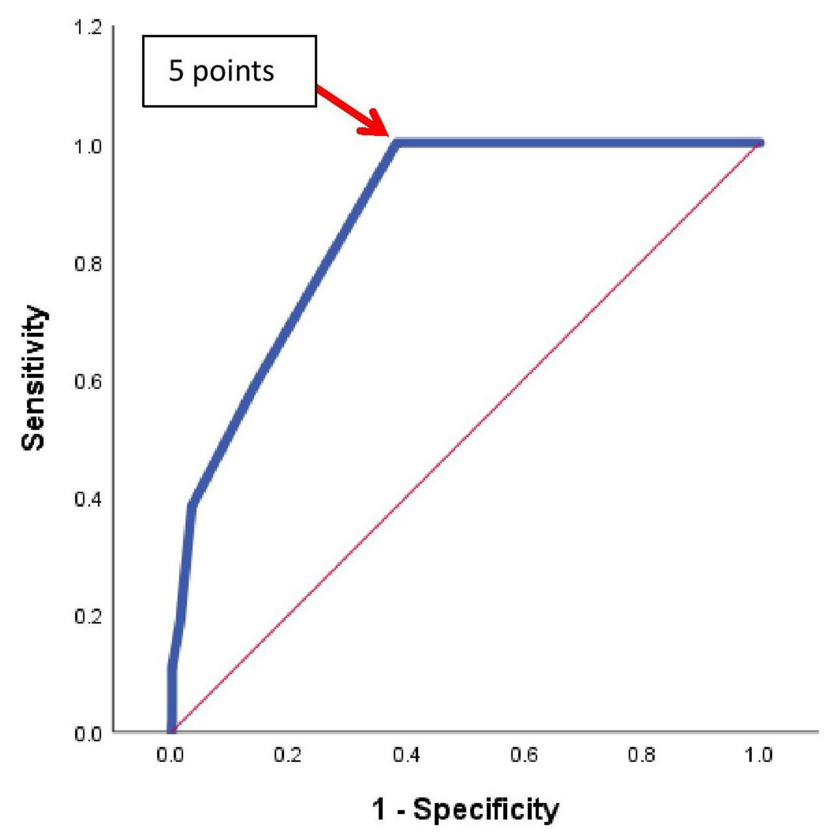

Figure I Receiver operating characteristic curve of the Revised Geneva Score cutoff for the exclusion of pulmonary embolism. [The cut of point of Revised Geneva score for diagnosis of pulmonary embolism is 5 points. At this point, the sensitivity is $100 \%$, specificity $=61.7 \%, \mathrm{PPV}=34.1 \%, \mathrm{NPV}=100 \%$ and $\mathrm{kappa}=0.35$, $\mathrm{p}=<0.001]$. was the optimum RGS cutoff for prediction of PE, with 100\% sensitivity, $61.7 \%$ specificity, $34.1 \%$ PPV and 100\% NPV, and an area under the curve of 0.87 . Douma et $\mathrm{al}^{24}$ have validated the RGS two category scale of $\leq 5$ and $>5$, on 807 patients with suspected PE and found only $1 \mathrm{PE}$ among 185 patients who were PE unlikely and had a normal dimer. According to the findings of our study, at this cutoff $<5, \mathrm{PE}$ could have been excluded even with an abnormal age-adjusted D-dimer threshold, with no missed PE cases. This would have saved the cost of $1036(51.5 \%)$ of all CTPAs. Of 1730 patients whose ageadjusted D-dimer threshold was abnormal, only 860 patients $(50 \%)$ could have been tested by CTPA, while for the remaining $870(50.2 \%)$ cases whose clinical probability was unlikely, PE could have been ruled out without further imaging. This finding was in agreement with the finding of a previous study that reported that using the RGS and a rule-out cut point of $\leq 5,62$ patients of 102

Table 5 Application of a Clinical Decision Rule and Age-Adjusted D-dimer Threshold for Prediction of PE

\begin{tabular}{|c|c|c|c|}
\hline $\begin{array}{l}\text { Age- } \\
\text { Adjusted } \\
\text { D-dimer }\end{array}$ & $\begin{array}{l}\text { Revised Geneva } \\
\text { Score }\end{array}$ & $\begin{array}{l}\text { No. of } \\
\text { Patients }\end{array}$ & $\begin{array}{l}\text { PE } \\
\text { Patients } \\
\text { No.(\%) }\end{array}$ \\
\hline \multirow[t]{2}{*}{ Abnormal } & Likely (>5points) & 860 & $308(35.8)$ \\
\hline & Unlikely ( $\leq 5$ points) & 870 & $0(0.0)$ \\
\hline \multirow[t]{2}{*}{ Normal } & Likely (>5points) & 114 & $24(21.1)$ \\
\hline & Unlikely ( $\leq 5$ points) & 166 & $0(0.0)$ \\
\hline
\end{tabular}

Abbreviation: PE, pulmonary embolism. 
patients $(60.8 \%)$ would have been spared CTPA at a cost of no PEs missed. ${ }^{15}$

Our study showed that both the conventional and ageadjusted thresholds had high sensitivity and low specificity, with a slightly increased specificity from $12.3 \%$ by conventional threshold to $15.3 \%$ by the age-adjusted threshold. This finding is in agreement with the findings of a retrospective study in Scotland, ${ }^{15}$ which reported an increased specificity from $11 \%$ to $24.9 \%$. In the present study, among patients aged 50 years or more, 8 and $12 \mathrm{PE}$ patients were missed, as false negatives, by the conventional and the age-adjusted thresholds, respectively. False-negative rates ranged from 0.0 to $0.6 \%$ using a conventional threshold, and $0.2-0.8 \%$ using an age-adjusted threshold. ${ }^{13,21,22}$ In our study, the corresponding rates were $1.0 \%$ and $1.2 \%$ for conventional and age-adjusted thresholds, respectively.

This study has some limitations. This study is based on data from one tertiary hospital in Saudi Arabia, and this may not allow for the generalization of the conclusion. Moreover, it is a retrospective study from patients' records and may make it liable to some information biases. Large-scale prospective studies, targeting the whole Saudi Arabia may be necessary.

\section{Conclusion}

The findings of this study confirm the fact that applying a clinical decision rule in combination with an age-adjusted D-dimer threshold would have increased the number of patients in whom PE could have been spared CTPA. PE would have been ruled out for more than one-half of all suspected cases if the Geneva score alone had been used. All those patients would have been spared CTPA with no cost of PEs missed. It may be safe to withhold oral anticoagulation therapy in patients with suspected PE, if RGS is low according to the 3 risk category scale, or unlikely ( $<5$ points) according to the 2 risk category scale.

\section{Data Sharing Statement}

Most of the data supporting our findings is contained within the manuscript, and all others, excluding identifying/confidential patient data should, will be shared upon request.

\section{Ethics Approval and Consent to Participate}

This study was approved by the institutional review board of the MNG-HA in Riyadh, Saudi Arabia (RC19/020/R). There was no need for consenting by patients as the study was done by retrieving data from medical records with no personal identifiers, and waiving of consent was approved by the IRB committee. This study was conducted in accordance with the Declaration of Helsinki.

\section{Acknowledgments}

This study was initiated by King Abdullah International Medical Research Center, King Saud bin Abdulaziz University for Health Sciences, Riyadh, Saudi Arabia. The final draft of the manuscript was English language edited by Macmillan Science Communication.

\section{Author Contributions}

All authors made substantial contributions to conception and design, acquisition of data, or analysis and interpretation of data; took part in drafting the article or revising it critically for important intellectual content; agreed to submit to the current journal; gave final approval of the version to be published; and agree to be accountable for all aspects of the work.

\section{Disclosure}

The authors declare that they have no competing interests.

\section{References}

1. Agnelli G, Becattini C. Acute pulmonary embolism. N Engl J Med. 2010;363(3):266-274. doi:10.1056/NEJMra0907731

2. Al-Hameed FM, Al-Dorzi HM, Al-Momen AM, et al. The Saudi Clinical Practice Guideline for the treatment of venous thromboembolism: outpatient versus inpatient management. Saudi Med J. 2015;36(8):1004.

3. Heit JA. The epidemiology of venous thromboembolism in the community: implications for prevention and management. In: The Vein Book. Academic Press; 2007:323-330.

4. Bĕlohlávek J, Dytrych V, Linhart A. Pulmonary embolism, part I: epidemiology, risk factors and risk stratification, pathophysiology, clinical presentation, diagnosis and nonthrombotic pulmonary embolism. Exp Clin Cardiol. 2013;18(2):129.

5. Thenappan T, Ormiston ML, Ryan JJ, Archer SL. Pulmonary arterial hypertension: pathogenesis and clinical management. $B M J$. 2018;360.

6. Le Gal G, Righini M, Roy PM, et al. Prediction of pulmonary embolism in the emergency department: the revised Geneva score. Ann Intern Med. 2006;144(3):165-171.

7. Wells PS, Anderson DR, Rodger M, et al. Derivation of a simple clinical model to categorize patients probability of pulmonary embolism: increasing the models utility with the SimpliRED D-dimer. Thromb Haemost. 2000;83(03):416-420.

8. Chong LY, Fenu E, Stansby G, Hodgkinson S. Management of venous thromboembolic diseases and the role of thrombophilia testing: summary of NICE guidance. BMJ. 2012;344:e3979.

9. Righini M, Goehring C, Bounameaux H, Perrier A. Effects of age on the performance of common diagnostic tests for pulmonary embolism. Am J Med. 2000;109(5):357-361.

10. Rumley A, Emberson JR, Wannamethee SG, Lennon L, Whincup PH, Lowe GD. Effects of older age on fibrin D-dimer, C-reactive protein, and other hemostatic and inflammatory variables in men aged 60-79 years. J Thromb Haemost. 2006;4(5):982-987. doi:10.1111/j.1538-7836.2006.01889.x 
11. Penaloza A, Kline J, Verschuren F, et al. European and American suspected and confirmed pulmonary embolism populations: comparison and analysis. J Thromb Haemost. 2012;10(3):375-381.

12. Wiener RS, Ouellette DR, Diamond E, et al. An official American Thoracic Society/American College of Chest Physicians policy statement: the Choosing Wisely top five list in adult pulmonary medicine. Chest. 2014;145(6):1383-1391.

13. Douma RA, Le Gal G, Söhne M, et al. Potential of an age adjusted D-dimer cut-off value to improve the exclusion of pulmonary embolism in older patients: a retrospective analysis of three large cohorts. BMJ. 2010;340(mar30 3):c1475. doi:10.1136/bmj.c1475

14. Fuchs E, Asakly S, Karban A, Tzoran I. Age-adjusted cutoff d-dimer level to rule out acute pulmonary embolism: a validation cohort study. Am J Med. 2016;129(8):872-878. doi:10.1016/j. amjmed.2016.02.043

15. Nobes J, Messow CM, Khan M, Hrobar P, Age-adjusted IC. D-dimer excludes pulmonary embolism and reduces unnecessary radiation exposure in older adults: retrospective study. Postgrad Med J. 2017;93(1101):420-424.

16. Jaconelli T, Eragat M, Crane S. Can an age-adjusted D-dimer level be adopted in managing venous thromboembolism in the emergency department? A retrospective cohort study. Eur J Emerg Med. 2018;25(4):288-294. doi:10.1097/MEJ.0000000000000448

17. Stein CE, Keijsers CJ, Bootsma JE, Schouten HJ. Missed diagnosis of pulmonary embolism with age-adjusted d-dimer cut-off value. Age Ageing. 2016;45(6):910-911.
18. Lapner ST, Stevens SM, Woller SC, Snow G, Kearon C. Ageadjusted versus clinical probability-adjusted D-dimer to exclude pulmonary embolism. Thromb Res. 2018;167:15-19. doi:10.1016/j. thromres.2018.05.003

19. Ten Cate-Hoek AJ, Prins MH. Management studies using a combination of D-dimer test result and clinical probability to rule out venous thromboembolism: a systematic review. J Thromb Haemost. 2005;3(11):2465-2470.

20. Klok FA, Kruisman E, Spaan J, et al. Comparison of the revised Geneva score with the Wells rule for assessing clinical probability of pulmonary embolism. J Thromb Haemost. 2008;6(1):40-44.

21. Penaloza A, Roy PM, Kline J, et al. Performance of age-adjusted Ddimer cut-off to rule out pulmonary embolism. J Thromb Haemost. 2012;10(7):1291-1296.

22. Righini M, Van Es J, Den Exter PL, et al. Age-adjusted D-dimer cutoff levels to rule out pulmonary embolism: the ADJUST-PE study. JAMA. 2014;311(11):1117-1124.

23. Pioped Investigators. Value of the ventilation/perfusion scan in acute pulmonary embolism. Results of the prospective investigation of pulmonary embolism diagnosis (PIOPED). JAMA. 1990;263 (20):2753.

24. Douma RA, Mos IC, Erkens PM, et al. Performance of 4 clinical decision rules in the diagnostic management of acute pulmonary embolism: a prospective cohort study. Ann Intern Med. 2011;154 (11):709-718.
International Journal of General Medicine

\section{Publish your work in this journal}

The International Journal of General Medicine is an international, peer-reviewed open-access journal that focuses on general and internal medicine, pathogenesis, epidemiology, diagnosis, monitoring and treatment protocols. The journal is characterized by the rapid reporting of reviews, original research and clinical studies

\section{Dovepress}

across all disease areas. The manuscript management system is completely online and includes a very quick and fair peer-review system, which is all easy to use. Visit http://www.dovepress.com/ testimonials.php to read real quotes from published authors. 